\title{
The correlation between serum cholesterol, asthma control and therapeutic step in children with asthma and aero allergenic sensitization
}

\author{
Ana-Maria Moiceanu Sovarel ${ }^{1,2}$, Eugenia Buzoianu ${ }^{1,2}$, \\ Mariana Moiceanu', Doina Anca Plesca ${ }^{1,2}$ \\ 1"Carol Davila" University of Medicine and Pharmacy, Bucharest, Romania \\ 2"Dr. Victor Gomoiu” Clinical Children's Hospital, Bucharest, Romania
}

\begin{abstract}
Asthma is a heterogeneous disease characterized by chronic inflammation of the airways. Cholesterol is a pro-inflammatory factor linked in some studies to asthma inflammation.

A prospective study has been started between January 2016 and September 2018 including 100 children aged 5 to 18 years old, diagnosed with allergic asthma and aero allergenic sensitization, evaluated and monitored in "Dr. Victor Gomoiu" Clinical Children's Hospital. All patients enrolled in the study were monitored for the level of cholesterol, the therapeutic step and the degree of asthma control.

The objective of the study was to assess the correlation between serum cholesterol and asthma control and between serum cholesterol and the step of controller treatment (as marker of asthma severity).

Conclusions. In the studied group, patients in the categories of partially controlled and uncontrolled asthma were in a higher percentage with higher values of serum cholesterol compared with those with controlled asthma; the children registered in the categories of moderate and severe asthma were in a higher percentage with total serum cholesterol increased compared to those with mild asthma.
\end{abstract}

Keywords: asthma, control, cholesterol, controller treatment

\section{INTRODUCTION}

Asthma is a heterogeneous disease characterized by chronic inflammation of the airways. Cholesterol is a pro-inflammatory factor linked in some studies to asthma inflammation $(1,2)$. Asthma is one of the most common chronic diseases in children and a major health problem around the world $(3,4)$.

Airway inflammation plays a leading role in asthma physiopathology alongside bronchial hyper reactivity $(3,4)$. Airway inflammation in asthma is correlated with genetic factors, age, favorable factors (passive smoking, exposure to pollutants, socio-economic status), trigger exposure (aeroallergens), controller treatment and asthma control.

The allergic asthma phenotype is found in most children with asthma and is registered in about 50\% of adults. The objective of allergenic sensitization to pollen of trees and grasses, animal epithelia, dust mites in house dust is achieved by determining immunoglobulin E antibodies and / or by prick skin tests (4).

There are significant differences among asthma patients regarding the difficulty in acquiring asthma control through successfully reducing bronchial inflammation. Many studies tried to identify the underlying cause of these differences regarding the therapeutic results.

According to some authors, the increased cholesterol levels in asthma patients may have a pro-inflammatory role $(3,5)$.

Hypercholesterolemia appears to be associated with increased expression of proinflammatory mechanisms, which would increase the level of proinflammatory cytokines, cell adhesion molecules, and other plasma proteins (ceruloplasmin, fibrinogen, or alpha 1 antitrypsin) involved in inflammatory sensitization $(3,4)$. 


\section{PURPOSE}

The objective of the study was to verify whether there is an association between:

- serum total cholesterol level and degree of disease control in children with allergic asthma and aeroallergenic sensitization;

- the serum total cholesterol level and the therapeutic control step (as a marker of asthma severity) in children with allergic asthma and aeroallergenic sensitization.

\section{AIM AND METHOD}

The prospective study included 100 children aged 5 to 18 years old, diagnosed with allergic asthma and aero allergenic sensitization, evaluated and monitored in "Dr. Victor Gomoiu" Clinical Children's Hospital between January 2016 and September 2018.

Inclusion criteria were: age between 5 and 18 years; asthma diagnosis based on clinical data and spirometry results (significant increase of FEV1 with $>12 \%$ or $200 \mathrm{ml}$ compared to the initial values after inhaled salbutamol); aero allergenic sensitization confirmed by increased specific serum IgE (immunoglobulin E) and/or positive prick skin tests.

Exclusion criteria were: non atopic asthma, other types of sensitization (food, drugs), familial dyslipidemia.

The study was approved by The Ethics Committee of the "Dr. Victor Gomoiu" Clinical Children's Hospital.

For each child the parents signed a written consent, ensuring confidentiality and protection of patient's identity.

Asthma control was evaluated in each patient based on daytime symptoms, nocturnal awakenings related to asthma, limitations of activity and use of salbutamol, according to the GINA criteria: well controlled, partially controlled or uncontrolled. In every case the controller therapy step was recorded (4).

For each patient the serum total cholesterol was measured and the result was interpreted in accordance with the National Cholesterol Education Program's (normal serum cholesterol is $\leq 200 \mathrm{mg} / \mathrm{dl}$ ).

Each patient received an individualized asthma action plan that included written recommendations for asthma management (6).

Finally we have assessed the association between:

- level of serum cholesterol and the degree of control of the asthma;

- level of serum cholesterol and the step of controller therapy.

\section{RESULTS AND DISCUSSIONS}

\section{The influence of the total cholesterol level on the degree of asthma control}

$44.7 \%$ of the pediatric patients included in the study had controlled asthma and a normal level of cholesterol, while $3.8 \%$ of the patients with controlled asthma had high cholesterol levels. Of the 100 asthmatic children included in the study, $10.6 \%$ had partially controlled asthma and a normal level of cholesterol while $43.4 \%$ of the patients with partially controlled asthma had an increased cholesterol level. $58.8 \%$ of those with uncontrolled asthma had high levels of serum cholesterol. In the table no. 1 shows the distribution of cholesterol values according to the degree of asthma control.

Using the chi square test to analyze the influence of serum cholesterol on the degree of asthma control (controlled, partially controlled and uncontrolled) resulted in a statistically significant association between the two variables $(p<0.001)$, meaning that a higher percentage of those with elevated cholesterol it falls into the categories of partially controlled and uncontrolled asthma compared to those of the controlled asthma category where the percentage of those with normal cholesterol is higher, as depicted in figure 1 .

\section{Influence of total cholesterol level on asthma severity}

In the patients who received "controller" therapy corresponding to step 1 or $2,25.5 \%$ had normal cho-

TABLE 1. Distribution of cases with normal values respectively high values of cholesterol in terms of the degree of asthma control

\begin{tabular}{|c|c|c|c|c|c|c|}
\hline & \multicolumn{3}{|c|}{ Degree of control } & \multirow{2}{*}{ Total } \\
\hline & & & Controlled & Partially controlled & Uncontrolled & \\
\hline \multirow{6}{*}{ 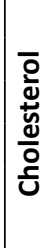 } & \multirow{2}{*}{ Normal } & Total & 21 & 5 & 21 & 47 \\
\hline & & $\%$ & $44.7 \%$ & $10.6 \%$ & $44.7 \%$ & $100.0 \%$ \\
\hline & \multirow{2}{*}{ Increased } & Total & 2 & 23 & 28 & 53 \\
\hline & & $\%$ & $3.8 \%$ & $43.4 \%$ & $52.8 \%$ & $100.0 \%$ \\
\hline & \multirow{2}{*}{ Total } & Total & 23 & 28 & 49 & 100 \\
\hline & & $\%$ & $23.0 \%$ & $28.0 \%$ & $49.0 \%$ & $100.0 \%$ \\
\hline
\end{tabular}




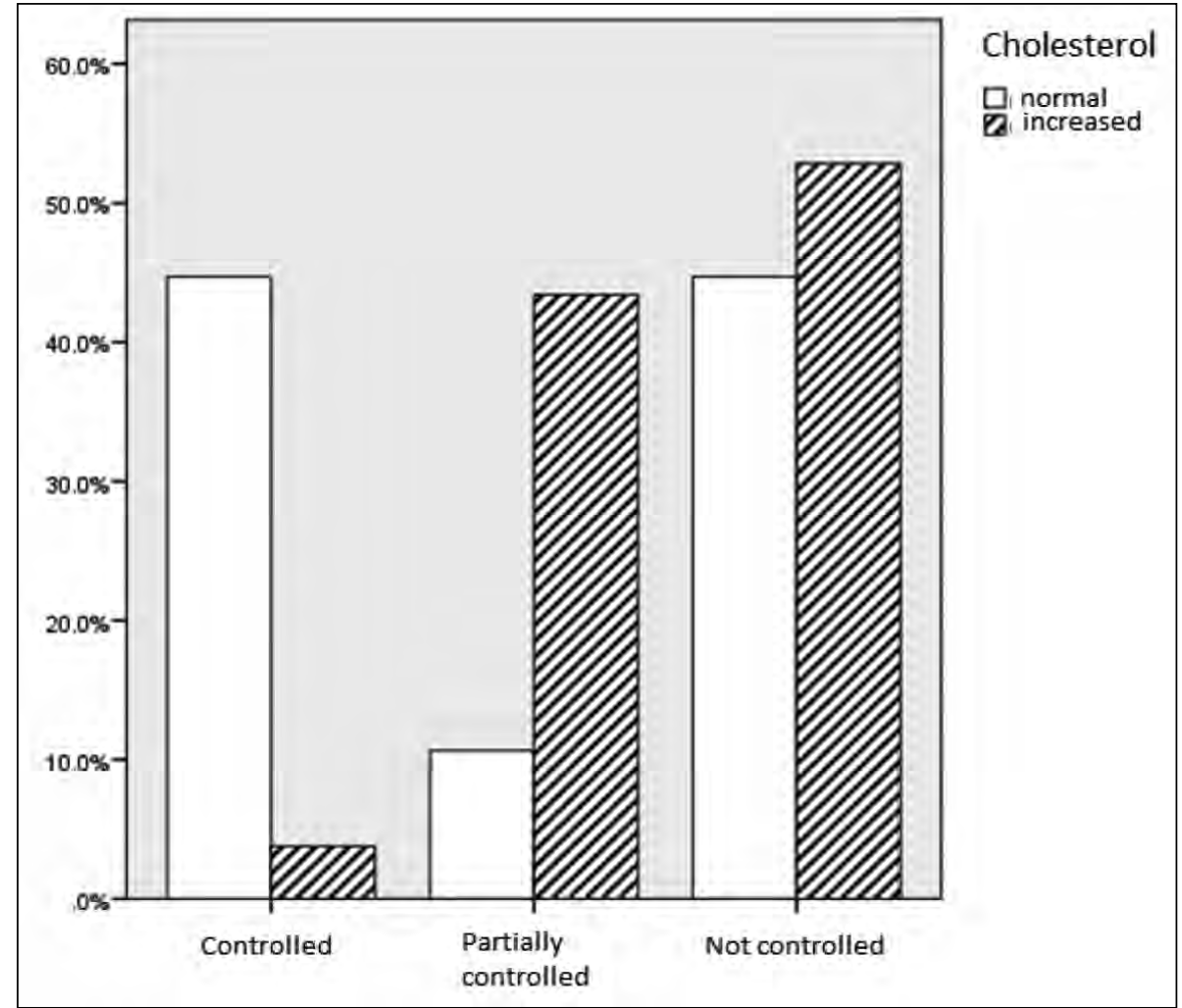

FIGURE 1. Distribution of the percentage of cases with total cholesterol, respectively increased on the three categories of the degree of asthma control lesterol values, while $9.4 \%$ of them had high cholesterol values.

$8.5 \%$ of patients receiving "controller" therapy according to step 4 had normal cholesterol, while $39.6 \%$ of them had elevated cholesterol. These results are summarized in table no.2.

TABLE 2. Distribution of cases with high respectively normal values of cholesterol in terms of therapeutic step

\begin{tabular}{|l|c|c|c|c|c|}
\hline \multicolumn{2}{|c|}{} & \multicolumn{3}{|c|}{ Therapeutic Step } & \multirow{2}{*}{ Total } \\
\cline { 3 - 6 } \multicolumn{2}{|c|}{} & $\mathbf{1}$ and 2 & $\mathbf{3}$ & $\mathbf{4}$ & \\
\hline \multirow{3}{*}{ Cholesterol } & Normal & 12 & 31 & 4 & 47 \\
\cline { 2 - 6 } & $\%$ & $25.5 \%$ & $66.0 \%$ & $8.5 \%$ & $100 \%$ \\
\cline { 2 - 6 } & High & 5 & 27 & 21 & 53 \\
\hline & $\%$ & $9.4 \%$ & $50.9 \%$ & $39.6 \%$ & $100 \%$ \\
\hline \multicolumn{2}{|l|}{ Total } & 17 & 58 & 25 & 100 \\
\hline
\end{tabular}

Using the Chi Square test to evaluate the influence of serum cholesterol level on the therapeutic step, we obtained a statistically significant correlation ( $p$ $<0.001$ ) in the sense that patients with high cholesterol fall in a higher percentage in categories 3 and 4 of the therapeutic step, as depicted in figure 2.

\section{DISCUSSIONS}

The proinflammatory role of serum cholesterol on the bronchial mucosa in asthma patients has aroused great scientific interest in recent years.
Existing research in the literature examining the link between cholesterol and asthma is mostly undertaken in adults and few in children, with conflicting results.

Thus, in a study by Karthikeyan Ramaraju et al, published in 2013, it was concluded that between asthma and serum cholesterol level there is a significant association, regardless of the patient's age, BMI or socio-economic level (3).

Rebecca K. Vinding et al. published in 2016 in "The Journal of Allergy and Clinical Immunology", volume 137, an article claiming that hypercholesterolemia tilts the adaptive immune system to the Th2 response that can affect some patients with diseases such as asthma (8).

In a meta-analysis published in March 2017 by $\mathrm{Su}$ $\mathrm{X}$ et al. on 10 clinical studies on the association between asthma and serum lipid profile, it was concluded that patients with asthma had a higher total cholesterol level compared to non-asthma patients. asthmatics (7).

The study of the association between blood lipid profile and asthma could open new perspectives on the management strategies of this disease in the future $(1,2,8)$. 


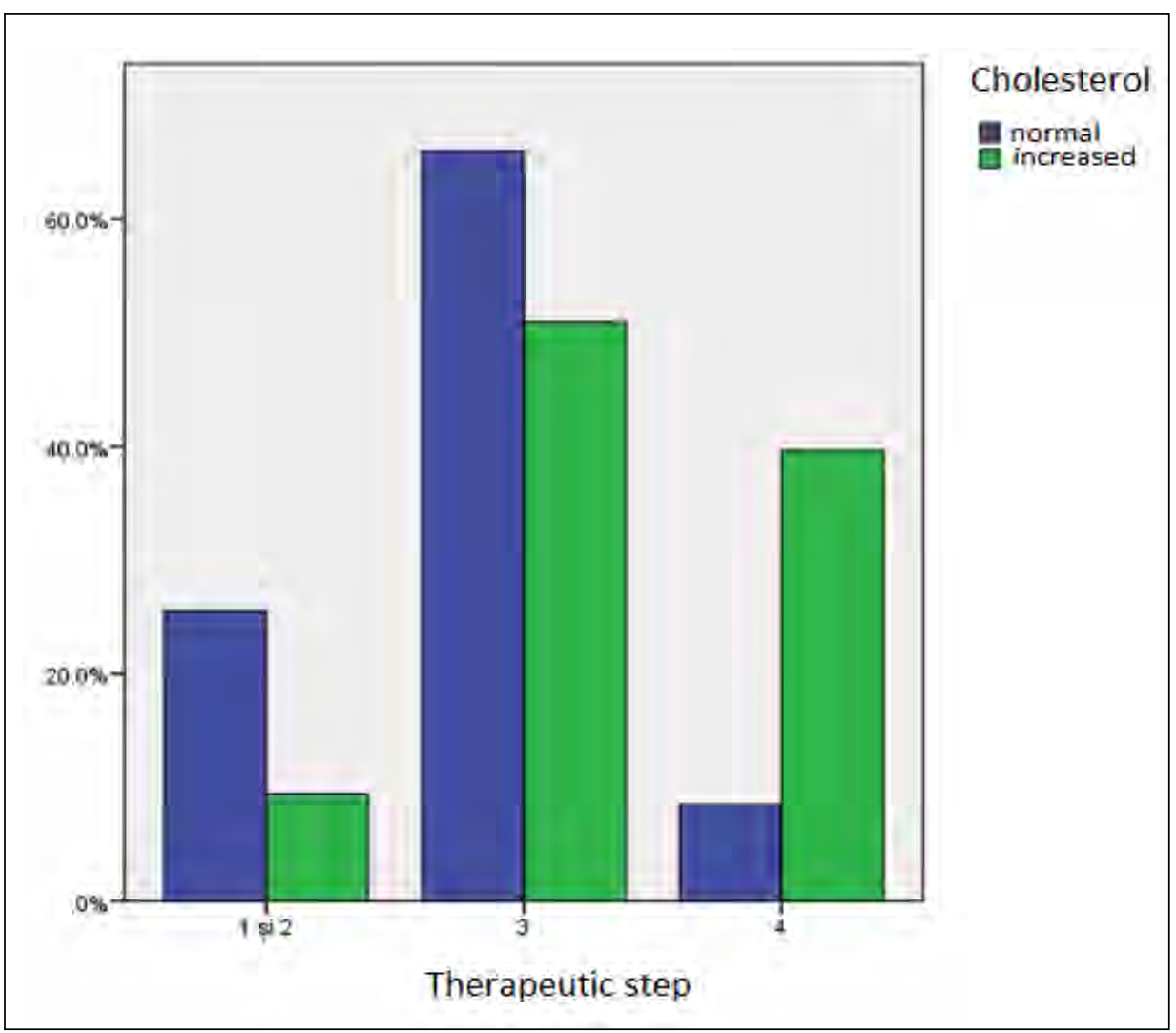

FIGURE 2. The distribution of the percentage of cases with respectively normal cholesterol increased respectively according to the therapeutic steps

\section{CONCLUSIONS}

In the studied group in children diagnosed with allergic asthma and aeroallergen sensitization serum cholesterol can be associated with the degree of disease control
In children diagnosed with allergic asthma and aeroallergen sensitization the level of serum cholesterol can be associated with their controller treatment step.

Conflict of interest: none declared Financial support: none declared

\section{REFERENCES}

1. Jaewook Jeong, Myong Ki Baeg, Sun-Hye Ko. Lipid profiles in adolescents with and without asthma: Korea National Health and nutrition examination survey data. Lipids Health Dis. 2018 July; Vol 17: pp 158

2. Chen YC, Su MW, Wang PC, Tsai CH, Tung KY. Lipid profiles in children with and without asthma: Interaction of asthma and obesity on hyperlipidemia. Diabetes Metabolism Syndrome 2013 Jan-Mar; Vol 7 (1): pp 20-5.

3. Ramaraju K, Krishnamurthy S, Maamidi S, Kaza AM, Balasubramaniam N. Is serum cholesterol a risk factor for asthma? Lung India 2013 Oct-Dec; Vol. 30, No.4, pp 295-301.

4. Lambrecht BN, Hammad H. The immunology of asthma. Nat Immunol. 2015 Jan; Vol. 16(1): pp 45-56.

5. Al-Shawwa B, Al-Huniti N, Titus G, Abu-Hasan M. Hypercholesterolemia is a potential risk factor for asthma. J Asthma. $2006 \mathrm{Apr}$; Vol 43(3): pp 231-3.

6. Global Strategy for Asthma Management and Prevention, Global Initiative for Asthma 2016.

7. Su X, Ren Y, Li M. Association between lipid profile and the prevalence of asthma: A meta-analysis and systemic review. Current Medical Research and Opinion. 2017 Nov 10: pp1-11.

8. Rebecca K Vinding, Jakob Stokholm. Blood lipid levels associate with childhood asthma, airway obstruction, bronchial hyperresponsiveness, and aeroallergen sensitization. The Journal of Allergy and Clinical Immunology 2016 January: Volume 137: pages 68-74. 